“(C) 2021 IEEE. Personal use of this material is permitted. Permission from IEEE must be obtained for all other uses, in any current or future media, including reprinting/republishing this material for advertising or promotional purposes, creating new collective works, for resale or redistribution to servers or lists, or reuse of any copyrighted component of this work in other works." 


\title{
A Composite Sliding Mode Control for SPMSM Drives Based on a New Hybrid Reaching Law with Disturbance Compensation
}

\author{
Xiaodong Sun, Senior Member, IEEE, Junhao Cao, Gang Lei, Member, IEEE, \\ Youguang Guo, Senior Member, IEEE, and Jianguo Zhu, Senior Member, IEEE
}

\begin{abstract}
This paper presents a composite sliding mode control (CSMC) method for speed control of surface-mounted permanent magnet synchronous motors (SPMSMs). The proposed CSMC consists of a new sliding mode control (SMC) based on a novel hybrid reaching law and an extended sliding mode disturbance observer (ESMDO). The new hybrid reaching law is composed of two parts, a terminal reaching part and an exponential plus proportional reaching part. It can effectively suppress the chattering and reduce the reaching time, compared with the conventional constant plus proportional rate reaching law (CPRL). The ESMDO is designed based on CPRL. It can estimate the extra chattering produced by the drive system's lumped disturbance and compensate for the controller's output. Based on the proposed new SMC and ESMDO, an antidisturbance sliding mode speed controller is designed to improve the performance of SPMSM drive systems. The performance of the proposed method has been validated experimentally and compared with the CPRL-based SMC methods under different conditions.
\end{abstract}

Keywords: Surface-mounted permanent magnet synchronous motors (SPMSM), sliding mode control, hybrid reaching law, disturbance observer.

\section{INTRODUCTION}

Due to the inherent advantages of high efficiency, high torque-inertia ratio and excellent control performance, permanent magnet synchronous motors (PMSMs) have been widely employed in many industrial applications, such as traction systems and robots [1]-[5]. PMSMs produce smaller torque ripples and noise compared with switched reluctance

Manuscript received Oct 11, 2020; revised Dec 4, 2020; accepted Jan 13, 2021. This work was supported by the National Natural Science Foundation of China under Project 51875261, the Natural Science Foundation of Jiangsu Province of China under Project BK20180046, the "Qinglan project" of Jiangsu Province, and the Postgraduate Research \& the Practice Innovation Program of Jiangsu Province under Project SJCX19_0574. (Corresponding author: Gang Lei)

$\mathrm{X}$. Sun and J. Cao are with the Automotive Engineering Research Institute, Jiangsu University, Zhenjiang 212013, China (email: xdsun@ujs.edu.cn, caojunhaoujs@163.com,).

G. Lei and Y. Guo are with the School of Electrical and Data Engineering, University of Technology Sydney, NSW 2007, Australia (e-mail: Gang.Lei@uts.edu.au, Youguang.Guo$1 @$ @uts.edu.au).

J. Zhu is with the School of Electrical and Information Engineering, University of Sydney, NSW, 2006, Australia (e-mail: jianguo.zhu@sydney.edu.au). machines [6]-[9]. To achieve good dynamic performance, several control schemes have been used to PMSMs, like fieldoriented control (FOC) [10]-[11].

However, the high sensibility of the controller performance to motor parameters entails the main challenge of the FOC scheme [12]. Therefore, the linear control methods like conventional proportional-integral (PI) control strategy will be difficult to provide a satisfactory control performance. Although PI regulators have the inherent ability to automatically compensate for modeling errors compared to predictive control systems, such compensation may not occur properly. To improve the system's robustness, nonlinear control methods have been investigated, such as robust control [13], adaptive control [14], predictive control [15],[16], state feedback control [17], [18], and sliding mode control (SMC). Among these nonlinear control schemes, the SMC strategy has been paid much attention.

SMC is a variable-structure control strategy which adopts a switching control law to alter the drive system's dynamics. It is insensitivity to parameter variations and holds fast dynamic response [19]-[21]. In [22], a fixed switching period SMC was proposed based on a decoupling method of the control actions, which could improve the PI-based FOC transient response. In [23], an improved direct instantaneous torque control based on adaptive terminal sliding mode control is proposed to suppress torque ripple and enhance the anti-interference ability for switched reluctance motors. In [24], a robust iterative learning control scheme was achieved by using an adaptive SMC technique, which could further reduce the torque ripples and improve the anti-disturbance ability of the motor servo system. An adaptive second order sliding mode observer with compensation of voltage source inverter (VSI) nonlinearity was applied to the sensorless servo system in [25]. The position estimation error can be reduced in wide-speed range while the voltage distortion can be compensated online to improve the model accuracy.

Usually, a large switching control gain of switching function is used to ensure the robustness of the SMC. However, the large switching control gain often causes serious chattering phenomenon [26]. To reduce the chattering level, saturation function is applied to substitute the switching function in the control law. Even though the chattering is reduced by this method, it produces uncertain steady-state error subject to the choice of the boundary layer [27]. Furthermore, some novel methods based on SMC strategy have been proposed to overcome this drawback, including the 
complementary sliding mode method [28], high order sliding mode method [29], and reaching law method [30].

Among these SMC strategies, owing to the direct association with the reaching process, the reaching law method can suppress the chattering significantly through a reasonable design of the reaching law. In [31], an SMC method based on a novel reaching law was introduced to reduce chattering on control input and maintain high tracking performance of the controller. In [32], a new switching type of reaching law for SMC of discrete-time system was proposed to ensure fast convergence and good robustness of the control system without increasing the magnitude of the critical signals. However, the complexity of the controller design entails challenge for its application in dynamical systems. To obtain disturbance observation of the control system, some methods based on extended sliding mode observations have been proposed. In [33], an anti-disturbance sliding mode observer was proposed and combined with sliding mode speed controller, which effectively reduces the torque ripples of the direct torque control of a four-phase switched reluctance motor. In [34], an extended sliding-mode mechanical parameter observer was presented to track system disturbances in real time, which includes the information of mechanical parameters. Furthermore, the equivalent lowpass filtering in the observer can be used to control chattering, thereby extra lowpass filtering can be avoided, and the output signal can be directly used for the parameter estimation. In [35], an extended state observer was designed based on a nonlinear model of electronic throttle to estimate the change of throttle opening angle and total disturbance. The estimation accuracy can be guaranteed by adjusting the gains parameters to change the convergent speed.

In this paper, a new SMC based on a novel hybrid reaching law (HRL) with disturbance observer is designed to suppress sliding chattering. It avoids the influence of switching function term in the constant plus proportional rate reaching law (CPRL) on system control performance. The hybrid reaching law contains two parts, a terminal reaching portion and exponential plus proportional rate reaching portion. In order to achieve real-time online estimation of system disturbance, a model-based extended sliding mode disturbance observer (ESMDO) is proposed to compensate the estimated perturbance to sliding mode speed control. Based on the analysis, a composite sliding mode control (CSMC) strategy consisting of the SMC and ESMDO is developed. The design of the controller combines the advantages of two reaching laws to realize global fast convergence. Further more, based on the mechanical equation, the observer can achieve fasttracking of global disturbance, which is effectively introduced in the structure of the control current to enhance the controller.

The remainder of this paper is organized as follows. Section II illustrates the mathematical model of surface-mounted permanent magnet synchronous motors (SPMSMs) and presents the FOC scheme. In Section III, the proposed HRL is presented, and its performance is analyzed by comparing it with the CPRL. Additionally, the stability of the proposed reaching law is proved. The design of the speed controller is detailed. Section IV presents model-based ESMDO to compensate the estimated perturbance and the structure of the CSMC scheme. Experimental results are provided in Section $\mathrm{V}$, followed by the conclusion.

\section{MATHEMATICAL MODEL AND FOC OF SPMSMS}

Assuming that the iron core of the SPMSMs is unsaturated and the eddy currents and hysteresis phenomenon are negligible, the mathematical model of the SPMSMs in the synchronous reference frame can be presented by

$$
\begin{gathered}
\left\{\begin{array}{l}
u_{d}=R i_{d}+\dot{\psi}_{d}-\omega_{e} \psi_{q} \\
u_{q}=R i_{q}+\dot{\psi}_{q}+\omega_{e} \psi_{d}
\end{array}\right. \\
\left\{\begin{array}{c}
\dot{\omega}_{m}=\frac{1}{J}\left(T_{e}-T_{L}-v \omega_{m}\right) \\
T_{e}=\frac{3}{2} p_{n} \psi_{f} i_{q}
\end{array}\right.
\end{gathered}
$$

The flux linkage equation is described as

$$
\left\{\begin{array}{l}
\psi_{d}=L i_{d}+\psi_{f} \\
\psi_{q}=L i_{q}
\end{array}\right.
$$

where $u_{d}, u_{q}$ and $i_{d}, i_{q}$ are the stator voltages and currents in $d$ $q$ coordinates, respectively. $R$ and $L$ represent the armature winding resistance and synchronous inductance, respectively. $\psi_{f}, J$, and $v$ are the permanent magnet flux linkage, rotor inertia and damping coefficient, respectively. $\omega_{m}$ is the rotor mechanical speed, $p_{n}$ is the number of pole pairs, and $\omega_{e}=$ $p_{n} \omega_{m}$ is the rotor electrical speed. $T_{e}$ is the electromagnetic torque, and $T_{L}$ is the load torque.

Fig. 1 shows a typical FOC block diagram for the control SPMSMs under no flux-weakening condition. The scheme adopts the structure of cascade control loops, including a speed loop and two current loops.

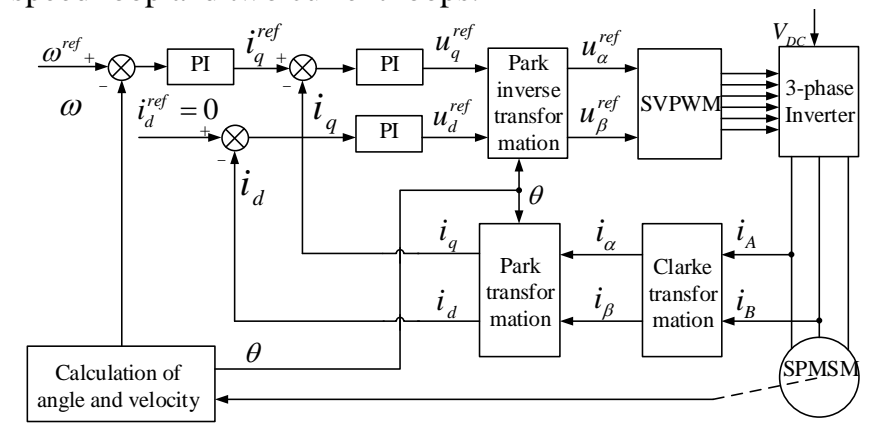

Fig. 1. FOC block diagram for SPMSMs under no flux-weakening condition.

\section{Sliding Mode SPeEd Controller Design}

3.1. SMC Design with Constant plus Proportional Rate Reaching Law (CPRL)

Compared with the general sliding mode variable structure control, the reaching law requires that the control system approaches the sliding surface to achieve the stability condition.

The basic form of the CPRL is expressed as follows

$$
\dot{s}=-\varepsilon \operatorname{sign}(s)-\lambda s \quad \lambda>0, \varepsilon>0
$$


where $s$ and sign () are the error between estimated and actual state variables (i.e., sliding mode variable) and sign function, respectively. $\varepsilon \operatorname{sign}(s)$ represents the isokinetic reaching term. Its reaching velocity in the approach motion stage is isokinetic, and the approach velocity can be changed by adjusting parameter $\varepsilon$. The approach velocity increases with the increase of the parameter $\varepsilon$ and vice verse. $\lambda s$ is the pure constant plus proportional rate term [36].

To illustrate the SMC theory, a second-order nonlinear system with the following state equation is considered as

$$
\left\{\begin{array}{l}
\dot{x}_{1}=x_{2} \\
\dot{x}_{2}=f(x)+b(x) u+d(x)
\end{array}\right.
$$

where $x=\left[x_{1}, x_{2}\right]$ the system state vector, $d(x)$ represents the system disturbances, and $f(x)$ and $b(x)$ are the bounded nonlinear matrix functions of the system states, respectively.

The sliding mode surface function of the system is defined as

$$
s=x_{1}+c x_{2}
$$

where parameter $c$ is the coefficient of the selected sliding mode switching surface, which will affect the convergence rate of the state variable after the system trajectory enters the sliding mode. Such a system sliding surface function ensures the accessibility of the sliding surface and the stability of the sliding mode motion. And the reaching rate is directly affected by the coefficient $c$.

Substituting (6) into (4) yields

$$
\dot{x}_{1}+c \dot{x}_{2}=-\varepsilon \operatorname{sign}(s)-\lambda s
$$

According (5) and (7), the control input can be obtained as follows

$$
u=-b(x)^{-1}\left[c^{-1}\left(x_{2}+\varepsilon \operatorname{sign}(s)+\lambda s\right)+f(x)+d(x)\right]
$$

Due to the sign function in control input, the chattering of the SMC is inevitable. And the chattering level is directly affected by the value of $\varepsilon$.

Considering that the system state trajectory moves from the $s>0$ side to the sliding mode surface, (4) can be simplified as

$$
\dot{s}=-\varepsilon-\lambda s
$$

The sliding mode variable function can be obtained by solving (9).

$$
s(t)=-\frac{\varepsilon}{\lambda}+\left[s(0)+\frac{\varepsilon}{\lambda}\right] e^{-\lambda t}
$$

where $s(0)$ represents the initial state of the sliding surface.

When the system state reaches the sliding surface, it satisfies $s(t)=0$. The reaching time can be calculated by simplifying (10) as

$$
t=\frac{1}{\lambda} \ln \left[1+s(0) \frac{\lambda}{\varepsilon}\right]
$$

According to the above formula, the reaching time of the constant plus proportional rate reaching law can be adjusted by the parameter $\lambda$. When the value of $\lambda$ is increased, the system robustness is improved while the approach speed is increased, but the increase of the value $\lambda$ will result in a corresponding increase in the chattering level of the control output.
Therefore, the reaching speed and the chattering level are contradictory in terms of the constant plus proportional rate reaching law. In order to solve the essential contradiction, a new reaching law is proposed based on the constant plus proportional rate reaching law.

\subsection{Proposed Hybrid Reaching Law (HRL)}

The novel HRL based on terminal reaching part control is proposed to further suppress the sliding chattering, which can avoid the influence of switching function term in conventional control law on system control performance. The reaching law is expressed as

$$
\dot{s}=-m|x|^{a} s^{q / p}-\frac{b}{k}\left(e^{k|x|}-1\right) s
$$

where $a>0, m>0, b>0$, and $0<k<1 . x$ is the system state variable. $p$ and $q$ are positive odd numbers and satisfy that $p$ is greater than $q$.

The hybrid reaching law consists of two parts, the terminal reaching part and the exponential plus proportional rate reaching part. The terminal reaching portion is represented by $-m|x|^{a} s^{q / p}$, which combines the power function of the system state variable on the basis of the terminal sliding mode to form a variable terminal approaching manner. The exponential plus proportional rate reaching part is represented by $-\frac{b}{k}\left(e^{k|x|}-1\right) s$.

This part introduces the exponential function of the system state variable based on the pure exponential reaching law, which produces a variable exponential reaching mode.

Since the system state variable is introduced in the reaching law, the sliding mode reaching speed is associated with the system state. When the state variable $|x|$ value is large (i.e., the SMC system trajectory is far away from the sliding mode switching surface). In the process of the system state approaching the sliding mode switching surface $s$, the terminal reaching part and the exponential plus proportional rate reaching part work simultaneously. The reaching coefficient is large enough to speed up the convergence process when the sliding mode switching surface is far away.

On the other hand, when the state variable $|x|$ is relatively small (i.e., the SMC system trajectory is close to the sliding mode switching surface), the reaching speed of the variable exponential plus proportional rate reaching portion approaches zero. Therefore, the system state approaches the sliding mode switching surface $s$ with the variable terminal sliding mode. The state variable $|x|$ enters the sliding mode switching surface and moves to the equilibrium point under the control output, which makes the reaching speed of the terminal reaching part decrease continuously. Thereby, the reaching speed is reduced when the system enters the sliding surface, and the chattering is effectively suppressed.

Summarily, in the case where the system state is relatively close to the sliding mode surface, the terminal reaching mode holds a fast reaching speed. The constant rate reaching mode approaches the sliding mode surface faster when the state trajectory is far away from the equilibrium point. The proposed hybrid reaching law combines the advantages of both to achieve global fast convergence. Furthermore, the 
power function of the system state variable is introduced in the reaching law, which suppresses the system chattering.

Referring to the derivation process in [27], the reaching time can be calculated from 0 to $t$ as

$$
t_{1}=\frac{k p}{b\left(e^{k|x|}-1\right)(p-q)} \ln \left[1+\frac{b\left(e^{k|x|}-1\right) s(0)^{\frac{p-q}{p}}}{k m|x|^{a}}\right]
$$

When the reaching speed of the hybrid reaching law is equal to the conventional exponential reaching law (i.e., $t=t_{1}$ ), it can be obtained that

$$
\left\{\begin{array}{l}
\frac{k p}{b\left(e^{k|x|}-1\right)(p-q)}=\frac{1}{\lambda} \\
\frac{b\left(e^{k|x|}-1\right) s(0)^{\frac{p-q}{p}}}{k m|x|^{a}}=s(0) \frac{\lambda}{\varepsilon}
\end{array}\right.
$$

Define $\frac{q}{p}$ as $\zeta$, which satisfies $0<\zeta<1$ The relationship of the parameters in the reaching laws can be simplified as

$$
\left\{\begin{array}{l}
\lambda=\frac{b}{k}\left(e^{k|x|}-1\right)(1-\zeta) \\
m(1-\zeta)|x|^{a} s(0)^{(1-\zeta)}
\end{array}\right.
$$

From (15), it can be further obtained that

$$
\begin{aligned}
& \frac{\lambda k}{b\left(e^{k|x|}-1\right)}=1-\zeta<1 \\
& \frac{\varepsilon}{m|x|^{a}}=(1-\zeta) \cdot s(0)^{(1-\zeta)}
\end{aligned}
$$

Therefore, the reaching coefficient of variable exponential reaching part in the proposed HRL is larger than the CPRL, which indicates that the variable exponential reaching part in the hybrid reaching law has the greater reaching speed.

Furthermore, the following inequality (18) can be obtained as

$$
\left\{\begin{array}{l}
\frac{\varepsilon}{m|x|^{a} s(t)^{(1-\zeta)}}>1, s(t)<s(0)(1-\zeta)^{-\frac{1}{\zeta}} \\
\frac{\varepsilon}{m|x|^{a} s(t)^{(1-\zeta)}} \leq 1, s(t) \geq s(0)(1-\zeta)^{-\frac{1}{\zeta}}
\end{array}\right.
$$

The terminal reaching part takes a major role when the system state is relatively close to the sliding surface, while the isokinetic reaching term is critical in the CPRL. It can be seen from (18) that the terminal reaching part in the proposed HRL takes smaller reaching speed compared with the isokinetic reaching term in the CPRL, which indicates the smaller chattering.

\subsection{Speed Control Law}

SMC aims to guarantee that the speed strictly follows its reference signal $\omega_{m}^{*}$ when the parameter changes in SPMSMs. Further, the basic idea of the sliding mode speed controller is to guide the state trajectory to progressively reach the sliding surface from any starting points by using the control law.
Therefore, the state variables of the system can be defined as

$$
\left\{\begin{array}{l}
x_{1}=\omega_{m}-\omega_{m}^{*} \\
x_{2}=\dot{\omega}_{m}
\end{array}\right.
$$

where $\omega_{m}^{*}$ is the reference mechanical speed, and $\omega_{m}$ is the actual output speed.

Substituting (2) into (19) yields

$$
x_{2}=\frac{1}{J}\left(\frac{3 p_{n} \psi_{f}}{2} i_{q}-T_{L}-v \omega_{m}\right)
$$

Derivation of (20) has the form as

$$
\dot{x}_{2}=\frac{1}{J}\left(\frac{3 p_{n} \psi_{f}}{2} \dot{i}_{q}-\dot{T}_{L}-v \dot{\omega}_{m}\right)=-\frac{v}{J} x_{2}+\frac{3 p_{n} \psi_{f}}{2 J} \dot{i}_{q}-\frac{\dot{T}_{L}}{J}
$$

Hence, the state equation of the system can be expressed as

$$
\left\{\begin{array}{l}
\dot{x}_{1}=x_{2} \\
\dot{x}_{2}=A x_{2}+B u+C
\end{array}\right.
$$

where $A=-\frac{v}{J}, B=\frac{3 p_{n} \psi_{f}}{2 J}, C=-\frac{T_{L}}{J}, u=i_{q}$.

Since the change of load disturbance $\dot{T}_{L}$ not considered, it satisfies $C=0$.

Select the linear sliding mode surface as follows.

$$
s=x_{2}+\eta x_{1}
$$

where $\eta$ is a constant.

Substituting (22) into the derivation of (23) yields

$$
\dot{s}=(A+\eta) x_{2}+B u
$$

Since the speed tracking error is a significant system performance indicator, it is selected as the state variable related to the reaching speed. Therefore, $x=x_{1}$ is substituted into (12), and the following equation can be obtained by combining (12) and (24).

$$
-m\left|x_{1}\right|^{a} s^{q / p}-\frac{b}{k}\left(e^{k\left|x_{1}\right|}-1\right) s=(A+\eta) x_{2}+B u
$$

Define $\zeta=q / p$, then the output of the controller can be obtained from (25) as

$$
u=\frac{1}{B}\left[-m\left|x_{1}\right|^{a} s^{\zeta}-\frac{b}{k}\left(e^{k\left|x_{1}\right|}-1\right) s-(A+\eta) x_{2}\right]
$$

Therefore, the reference $q$-axis current is written as

$$
i_{q}^{r e f}=\frac{1}{B} \int\left[-m\left|x_{1}\right|^{a} s^{\zeta}-\frac{b}{k}\left(e^{k\left|x_{1}\right|}-1\right) s-(A+\eta) x_{2}\right] d t
$$

The flowchart of the variable structure of the system parameter $i_{q}^{\text {ref }}$ is illustrated in Fig. 2.

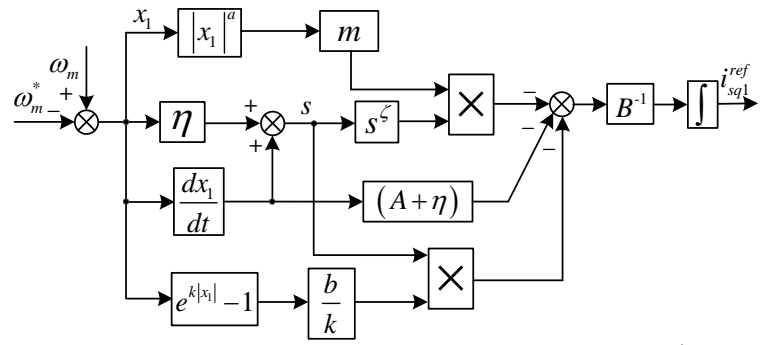

Fig. 2. Flow chart of the variable structure of system parameter $i_{q}^{\text {ref }}$. 


\section{Design of SLiding Mode Disturbance ObSERVER}

\subsection{Extended Sliding Mode Disturbance Observer (ESMDO)}

There are various disturbances in SPMSM drive systems under complex operating conditions. If these disturbances are not effectively suppressed, the system control performance will be affected. Therefore, to realize real-time online estimation of system disturbances, this paper proposes an ESMDO.

When the system disturbances are taken into consideration, (19) can be rewritten as

$$
\begin{aligned}
x_{2}^{*} & =\frac{3 p_{n} \psi_{f}}{2(J+\Delta J)} i_{q}-\frac{T_{L}+\Delta T_{L}}{J+\Delta J}-\frac{v+\Delta v}{J+\Delta J} \omega_{m} \\
& =\left(\frac{3 p_{n} \psi_{f}}{2 J}-\Delta_{1}\right) i_{q}-\left(\frac{1}{J}-\Delta_{2}\right)\left(T_{L}+\Delta T_{L}\right)-\left(\frac{v}{J}-\Delta_{3}\right) \omega_{m} \\
& =x_{2}-\Delta_{1} i_{q}-\left(\frac{1}{J}-\Delta_{2}\right) T_{L}+\Delta_{2} \cdot \Delta T_{L}+\Delta_{3} \omega_{m}
\end{aligned}
$$

where $\Delta J, \Delta v$ and $\Delta T_{L}$ represent the perturbance of the corresponding mechanical parameters, respectively. For the sake of simplicity, $\Delta_{1}, \Delta_{2}$, and $\Delta_{3}$ indicate the uncertainties.

The disturbances of the SPMSM drive systems consisting of mechanical parameter disturbance and load disturbance, which can be expressed as

$$
D=-\Delta_{1} i_{q}-\left(\frac{1}{J}-\Delta_{2}\right) \Delta T_{L}+\Delta_{2} \cdot \Delta T_{L}+\Delta_{3} \omega_{m}
$$

The comprehensive disturbance should satisfy the following constraints

$$
\left\{\begin{array}{l}
|D| \leq \bar{D} \\
\dot{D}=d
\end{array}\right.
$$

where $\bar{D}$ is the limit value of the comprehensive disturbance of the motor system and $d$ represents the variation rate of the comprehensive disturbance.

Taking the comprehensive perturbance into consideration, (22) is written as

$$
\left\{\begin{array}{l}
\dot{x}_{1}=x_{2} \\
\dot{x}_{2}=A x_{2}+B u+C+\dot{D}
\end{array}\right.
$$

Then, the reference $q$-axis current can be obtained as

$$
i_{q}^{r e f}=\frac{1}{B} \int\left[-m\left|x_{1}\right|^{a} s^{\zeta}-\frac{b}{k}\left(e^{k\left|x_{1}\right|}-1\right) s-(A+\eta) x_{2}-C-\dot{D}\right] d t
$$

From (32), it can be observed that the comprehensive disturbance is of significant effect on the control performance. If the system disturbance is negative and considered as 0 , the parameters $m$ and $b$ may increase to generate the same control output $i_{q}^{\text {ref }}$, which leads to severe system chattering.

Using $D$ as the extended state variable of the system, combined with (30) and (31), the extended state equation of the system can be obtained as

$$
\left\{\begin{array}{l}
\dot{\omega}_{m}=-\frac{v}{J} \omega_{m}+D+B i_{q}-\frac{T_{L}}{J} \\
\dot{D}=d
\end{array}\right.
$$

The sliding mode disturbance observer is constructed as

$$
\left\{\begin{array}{l}
\dot{\hat{\omega}}_{m}=-\frac{v}{J} \hat{\omega}_{m}+\hat{D}+B i_{q}-\frac{T_{L}}{J}+y \\
\dot{\hat{D}}=r y
\end{array}\right.
$$

where $\hat{\omega}_{m}$ and $\hat{D}$ represent the estimations of the speed and perturbance, respectively, $r$ is the control law gain coefficient, and $y$ is the observer function to ensure that speed error converges to 0 . The schematic diagram of the ESMDO is shown in Fig. 3.

According to (33) and (34), the error equation of the observer can be written as

$$
\left\{\begin{array}{l}
\dot{e}_{\omega}=-\frac{v}{J} e_{\omega}+e_{D}-y \\
\dot{e}_{D}=d-r y
\end{array}\right.
$$

where $e_{\omega}$ and $e_{D}$ are the errors of speed and disturbance observation, respectively.

The CPRL is employed to ensure that the sliding mode surface is chosen as $s \omega=e \omega$. Therefore, it can be obtained as

$$
\dot{s}_{\omega}=\dot{e}_{\omega}=-\varepsilon \operatorname{sign}\left(s_{\omega}\right)-\lambda s_{\omega}
$$

Substituting (36) into (35) yields

$$
y=-\frac{v}{J} e_{\omega}+e_{D}+\varepsilon \operatorname{sign}\left(s_{\omega}\right)+\lambda s_{\omega}
$$

Therefore, the observer with the control law $y$ can reach the sliding mode surface in finite time. Then, the following equations can be obtained as

$$
\left\{\begin{array}{l}
\dot{s}_{\omega}=s_{\omega}=0 \\
\dot{e}_{\omega}=e_{\omega}=0
\end{array}\right.
$$

Substituting (38) into the error (35) yields

$$
\left\{\begin{array}{l}
e_{D}-y=0 \\
d-r y=\dot{e}_{D}
\end{array}\right.
$$

From (39), the differential equation is obtained as follows

$$
\dot{e}_{D}+r e_{D}=d
$$

Solving (40), the disturbance estimation error can be obtained as

$$
e_{D}=e^{-r t}\left(K+\int d \cdot e^{r t} d t\right)
$$

where $K$ is a constant. Similarly, to ensure that the disturbance error converges to 0 , it must be satisfied that

$$
r>0
$$

Furthermore, the convergence speed of sliding mode disturbance observer is directly determined by the value of $r$.

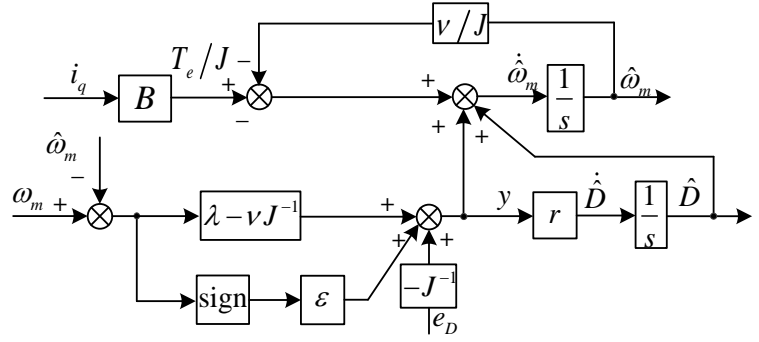

Fig. 3. Schematic diagram of the ESMDO.

\subsection{Proof of Stability}

In order to verify the stability of the control system with the proposed observer, the Lyapunov function $V=\mathrm{s}^{2} / 2$ is selected. 
When the inequality (42) is established, the sliding mode reaching condition is satisfied, and the system is stable.

$$
\dot{V}=s \dot{s} \leq 0
$$

Substituting (31) into (43) yields

$$
\begin{aligned}
\dot{V} & =s \dot{s}=s\left[(A+\eta) x_{2}+B u+C+\dot{D}\right] \\
& =-m|x|^{a} s^{\frac{p+q}{p}}-\frac{b}{k}\left(e^{k|x|}-1\right) s^{2}
\end{aligned}
$$

Since the parameter $p+q$ is even, the first and second terms in (44) are negative. It can be obtained that (43) is satisfied, which indicates the proposed HRL can ensure that the system motion reaches the sliding mode switching surface. Once the system state reaches and remains on the sliding mode switching surface, it enters the sliding mode.

\subsection{Anti-disturbance Sliding Mode Speed Controller}

In order to improve the performance of the SMC system under disturbances, the ESMDO is employed to estimate lumped disturbance. The disturbance term $D$ in the sliding mode speed control law is substituted as $\hat{D}$. Fig. 4 illustrates a structure of the anti-disturbance sliding mode controller based on the proposed ESMDO. Fig. 5 shows a block diagram of the CSMC strategy for SPMSM based on FOC. It consists of the SMC based on the HRL and the ESMDO.

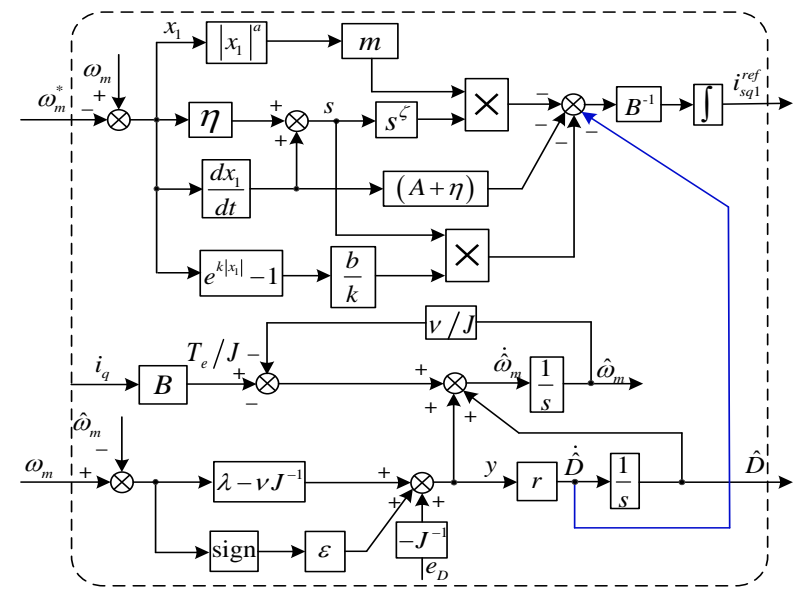

Fig. 4. Structure of the sliding mode controller based on the proposed ESMDO.

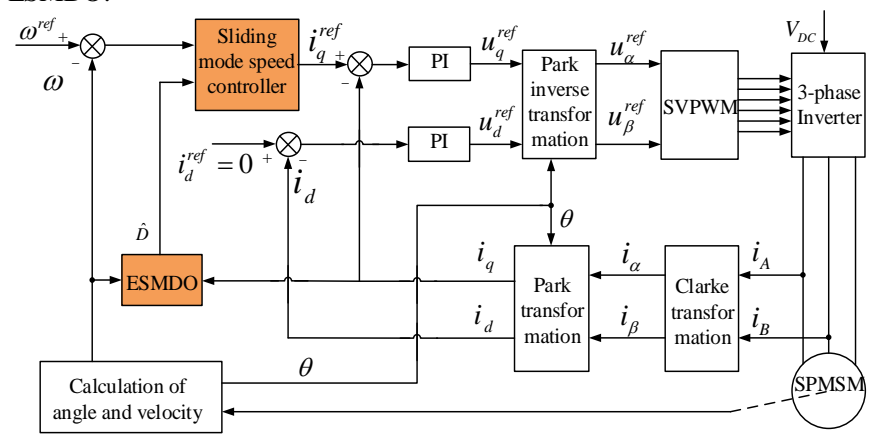

Fig. 5. Block diagram of the composite SMC strategy (CSMC) for SPMSM based on the FOC scheme.

As shown, the entire control system adopts a vector control series double closed-loop structure. The outer loop of velocity adopts a sliding mode velocity controller structure based on the novel SMC method. It combines the ESMDO to update the disturbance amount in real-time to form a CSMC strategy. The
ESMDO observes the disturbance in real-time and inputs the observation feedforward into the sliding mode speed controller to compensate the system disturbance, thereby improving the system's anti-disturbance capability.

\section{EXPERIMENTAL RESULTS}

To verify the system's control performance based on the proposed CSMC method, experiments have been conducted at a laboratory platform. The experimental setup consists of an SPMSM, a torque sensor, and a magnetic powder brake, as shown in Fig. 6. The proposed control strategy is carried out in a dSPACE 1401 test bench, through which the experimental measurements can be exported to MATLAB and plotted. Table I lists the main system parameters.

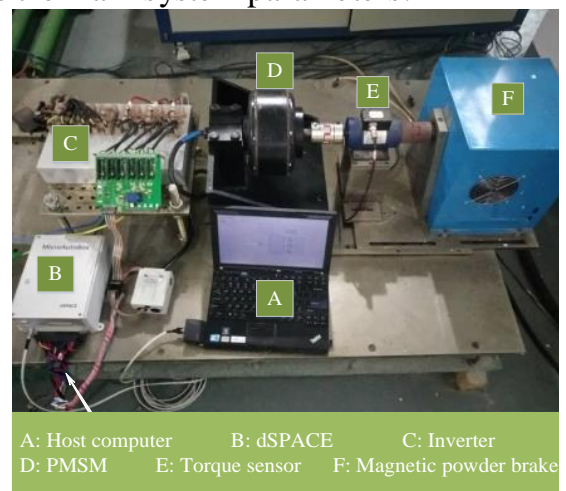

Fig. 6. Experimental setup.

The parameter selection of the proposed sliding mode controller includes the following principles. The parameters $a$ and $k$ should be selected based on the principle of chatter suppression while taking into account the reaching time; The parameter $\eta$ is the coefficient of the selected sliding mode switching surface, which will affect the convergence rate of the state variable after the system trajectory enters the sliding mode. The parameters $m$ and $b$ are the coefficients of terminal approaching mode and exponential plus proportional approaching mode, respectively. Meanwhile, the parameters should not be too large; otherwise, sliding mode chattering will occur. Thus, The parameters $p$ and $q$ will affect the convergence time of the terminal reaching law. The parameters of the CPRL-based SMC are: $c=20, \varepsilon=2, \lambda=$ 1300. The parameters of the CSMC strategy are: $m=1000, a$ $=0.2, q=1, p=3, b=950, k=1, \eta=c=20$.

Table I. System Parameters of the SPMSM

\begin{tabular}{ccc}
\hline \hline Parameters & Symbol & Value \\
\hline Number of pole pairs & $P_{n}$ & 22 \\
Stator resistance & $R$ & $0.08 \Omega$ \\
Stator inductance & $L$ & $4.2 \mathrm{mH}$ \\
Permanent- magnet flux linkage & $\psi_{f}$ & $0.625 \mathrm{~Wb}$ \\
Inertia & $J$ & $0.004 \mathrm{kgm}$ \\
Viscous friction coefficient & $v$ & $0.0006 \mathrm{~N} \cdot \mathrm{m} \cdot \mathrm{s}$ \\
Rated speed & $N$ & $360 \mathrm{rpm}$ \\
Rated power & $P_{N}$ & $30 \mathrm{~kW}$ \\
DC-link voltage & $u_{d c}$ & $420 \mathrm{~V}$ \\
\hline \hline
\end{tabular}

5.1. Performance Comparison under a Step Increase of Load Torque

Fig. 7 shows the dynamic responses of three control schemes under a step increase of load torque $T_{L}$ for the 
SPMSM drive system. The reference speed is $360 \mathrm{rpm}$, and the sampling time is set as $0.1 \mathrm{~ms}$. A step change of the load torque from 0 to $10 \mathrm{Nm}$ is applied to the control system at $t$ $=0.5 \mathrm{~s}$. Compared with the CPRL-based SMC control method, the proposed HRL method maintains better dynamic performance. It can be observed that the HRL method produces smaller speed overshoot when the load torque changes. Meanwhile, the HRL method produces smaller fluctuation than the CPRL method. Furthermore, the proposed CSMC with extended disturbance observer shows better performance in terms of speed tracking and torque. The specific comparative results of these three control schemes are listed in Table II.

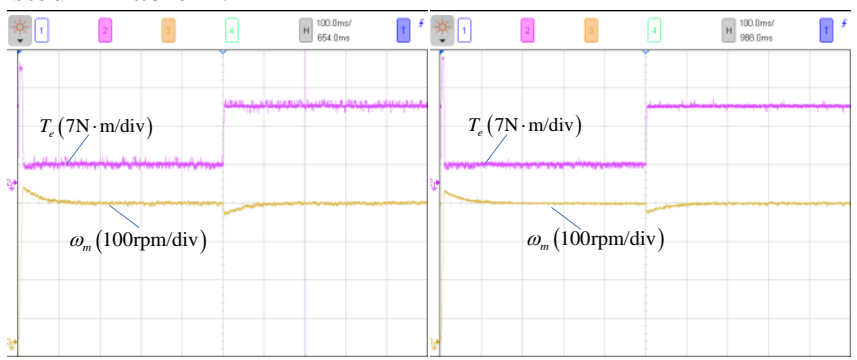

(a)

(b)

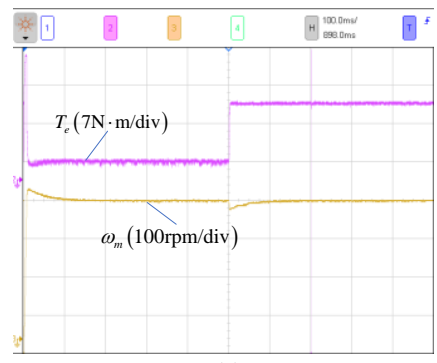

(c)

Fig. 7. Experimental performance under load torque step increase, (a) conventional CPRL-based method, (b) proposed HRL-based SMC method, and (c) the proposed CSMC method.

Table II. Performance Parameters of the Three Control Schemes under an Increase of Load Torque

\begin{tabular}{|c|c|c|c|}
\hline & $\begin{array}{c}\text { CPRL } \\
\text { method }\end{array}$ & $\begin{array}{c}\text { HRL } \\
\text { method }\end{array}$ & $\begin{array}{c}\text { CSMC } \\
\text { (CPRL+ESMDO) }\end{array}$ \\
\hline $\begin{array}{c}\text { Speed } \\
\text { fluctuation (rpm) }\end{array}$ & 10 & 7.8 & 5.4 \\
\hline $\begin{array}{c}\text { Torque } \\
\text { fluctuation (Nm) }\end{array}$ & 1.05 & 0.83 & 0.72 \\
\hline Settling time (s) & 0.013 & 0.011 & 0.010 \\
\hline
\end{tabular}

Fig. 8 shows the performance of the proposed ESMDO under load torque increase condition. As shown, with the increase of the load torque at $t=0.5 \mathrm{~s}$, the SMC law $y$ produces a fluctuation of $-1700 \mathrm{rad} / \mathrm{s}^{-2}$. The disturbance estimation value $\hat{D}$ changes stepwise to about $-2300 \mathrm{rad} / \mathrm{s}^{-2}$, which is mainly caused by load change. From Figs. 8(c) and (d), the error of the speed observation is small. Its maximum is $-1.6 \mathrm{rad} / \mathrm{s}$ when the step load torque is applied to the system. Therefore, the proposed ESMDO performs well in the presence of disturbance and speed observation, which contributes to the anti-disturbance sliding mode speed control.
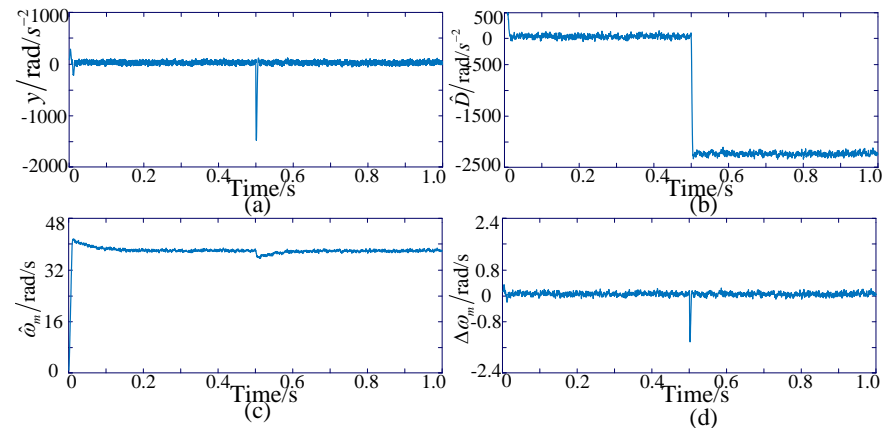

Fig. 8. ESMDO performance under load torque step increase condition: (a) SMC law of the ESMDO, (b) disturbance estimation value, (c) mechanical speed estimation value, and (c) speed observation error.

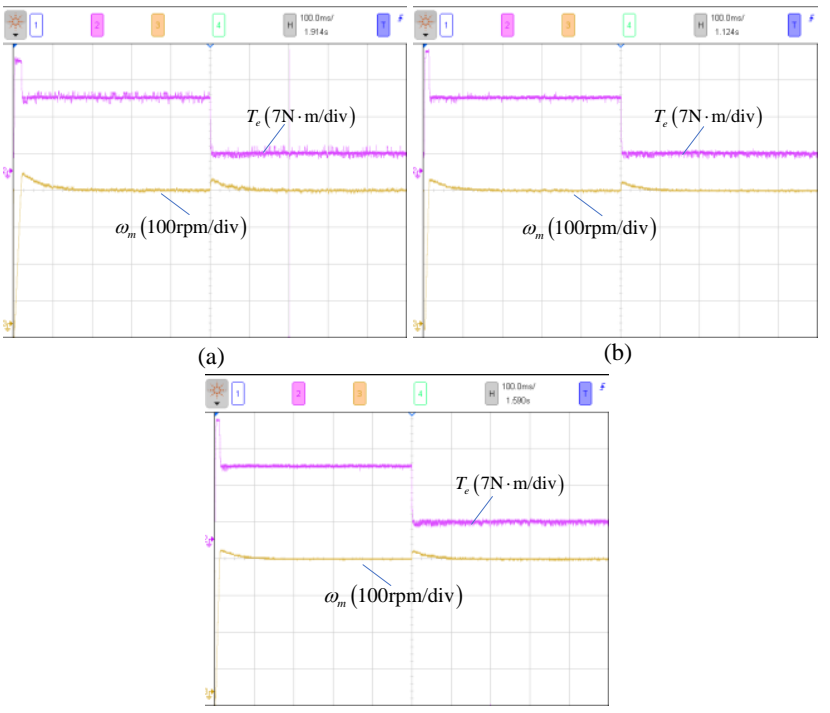

(c)

Fig. 9. Experimental performance under load torque step decrease condition: (a) CPRL-based SMC method, (b) proposed HRL-based SMC method, and (c) proposed CSMC method.

\subsection{Performance Comparison under a Step Decrease of Load} Torque

Fig. 9 illustrates the dynamic responses of three control schemes under a step decrease of load torque $T_{L}$ for the SPMSM drive system. A step-change in the reference torque from 10 to $0 \mathrm{Nm}$ is applied to the control system at $t=0.5 \mathrm{~s}$. Compared with the CPRL method, the proposed HRL method performs better in dynamic performance as well.

Fig. 10 shows the performance of the proposed ESMDO in this scenario. As shown, the step change of disturbance estimation value $\hat{D}$ is from -2300 to $0 \mathrm{rad} / \mathrm{s}^{-2}$, which is mainly determined by the second term $-\left(\frac{1}{J}-\Delta_{2}\right) \Delta T_{L}$ of the lumped disturbance. Therefore, the observation can estimate the disturbance exactly and quickly with small chattering, and the observation error of the rotational speed further verifies the performance of the observer. 

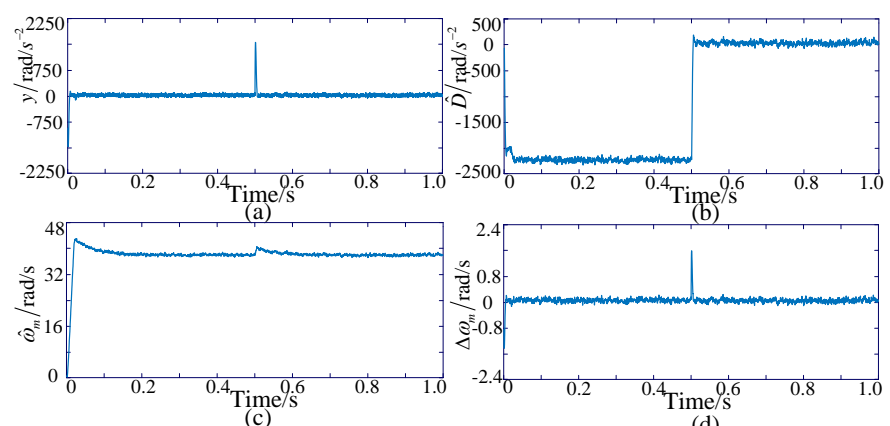

(d)

Fig. 10. ESMDO performance under load torque step decrease condition: (a) SMC law of the ESMDO, (b) disturbance estimation value, (c) mechanical speed estimation value, and (d) speed observation error.
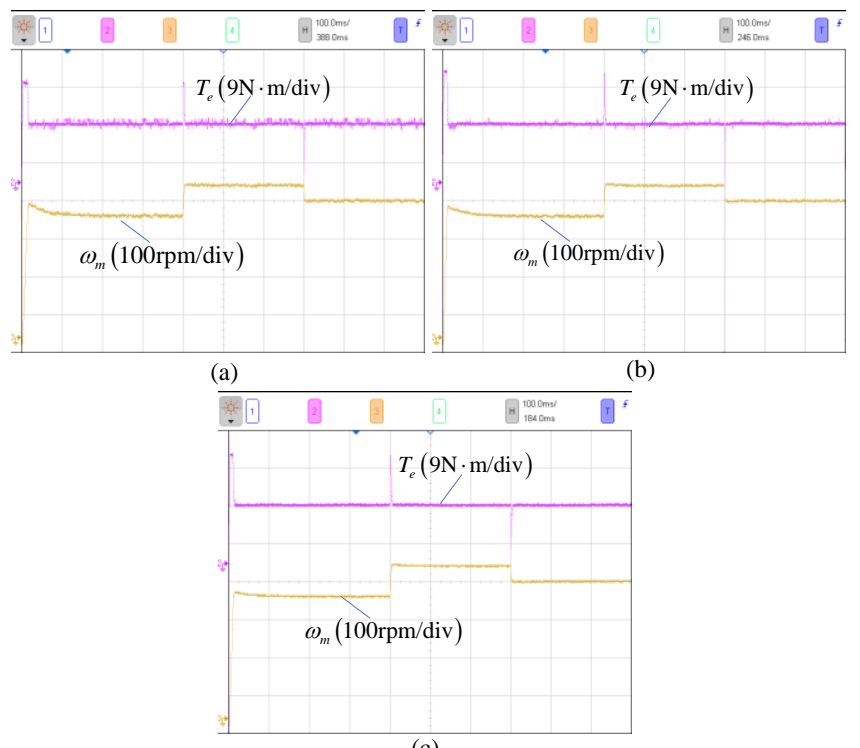

(c)

Fig. 11. Experimental performance under reference speed step-change condition: (a) CPRL-based SMC method, (b) proposed HRL-based SMC method, and (c) proposed CSMC method.

\subsection{Performance Comparison Under Step Changes to the Reference Speed}

Fig. 11 illustrates the dynamic responses of three control methods when step changes are applied to the reference mechanical speed. Two step-changes, 320 to $400 \mathrm{rpm}$ and 400 to $360 \mathrm{rpm}$, are applied to the system at the time instants $t_{1}=0.4 \mathrm{~s}$ and $t_{2}=0.7 \mathrm{~s}$, respectively. The load torque is set to 10 $\mathrm{Nm}$. As shown, the proposed HRL-based SMC method presents better dynamic performance than the SMC scheme based CPRL. The fluctuation in the mechanical speed $\omega_{m}$ and load torque $T_{L}$ is reduced with the HRL method. Furthermore, the HRL method shows a faster response in speed tracking.

As shown in Fig. 12, the ESMDO performs well in disturbance estimation, and the CSMC scheme presents the ability of disturbance suppression compared with CPRL method. The oscillations in the estimated disturbance $\hat{D}$ converge to zero in $0.02 \mathrm{~s}$, and the transient-state error of the mechanical speed is kept within $0.5 \mathrm{rad} / \mathrm{s}$.
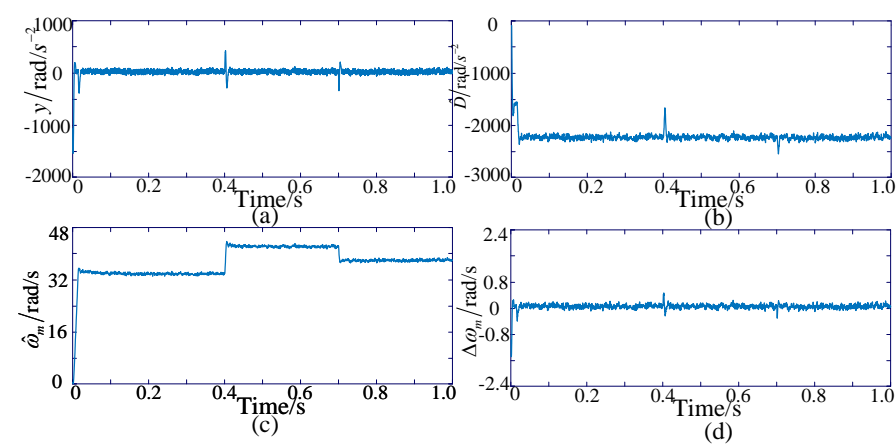

(d)

Fig. 12. ESMDO performance under reference speed step-change condition: (a) SMC law of the ESMDO, (b) disturbance estimation value, (c) mechanical speed estimation value, and (c) speed observation error.

\section{CONCLUSION}

A CSMC method composed of a novel HRL and an ESMDO was presented in this paper. The chattering of the sliding mode speed controller can be significantly suppressed by introducing a hybrid reaching law into the SMC. Compared with the CPRL, it was verified that the new reaching law yields smaller chattering and performs better dynamic response. In order to estimate system disturbance online, the ESMDO based on constant plus proportional rate reaching law was introduced. Furthermore, considering the uncertainty of motor parameters, the stability of the ESMDO was proved by solving the differential equation when the state variable of disturbance reaches the sliding mode surface. It can be concluded that the proposed ESMDO performs well in the presence of disturbance and speed observation, which contributes to the anti-disturbance sliding mode speed control.

The composite sliding mode speed controller has the advantages of reducing the speed and torque fluctuation under various conditions. Therefore the CSMC scheme presents the ability of disturbance suppression compared with CPRL method and the oscillations in the estimated disturbance converge to zero quickly. Due to the complexity of the new reaching law, the design of the sliding mode controller is more complicated than the traditional constant plus proportional law. The computation load of the whole control system will increase as well due to the introduction of the disturbance observer. In addition, for the sliding mode observer, the performance of the observer needs to be further studied for more accurate and fast observations.

\section{REFERENCES}

[1] M. Khayamy and H. Chaoui, "Current sensorless MTPA operation of interior PMSM drives for vehicular applications," IEEE Trans. Veh. Technol., vol. 67, no. 8, pp. 6872-6881, Aug. 2018.

[2] X. Sun, Z. Shi, G. Lei, Y. Guo, and J. Zhu, "Multi-objective design optimization of an IPMSM based on multilevel strategy," IEEE Trans. Ind. Electron., vol. 68, no. 1, pp. 139-148, Jan. 2021.

[3] J. Zheng, W. Zhao, J. Ji, J. Zhu and C. H. T. Lee, "Sleeve design of permanent-magnet machine for low rotor losses," Chinese Journal of Electrical Engineering, vol. 6, no. 4, pp. 86-96, Dec. 2020.

[4] Z. Shi, et al, "Robust design optimization of a five-phase PM hub motor for fault-tolerant operation based on Taguchi method," IEEE Trans. Energy Convers., vol. 35, no. 4, pp. 2036-2044. Dec. 2020.

[5] S. Song, G. Fang, R. Hei, J. Jiang, R. Ma and W. Liu, "Torque ripple and efficiency online optimization of switched reluctance machine based on torque per ampere characteristics," IEEE Trans. Power Electron., vol. 35, no. 9, pp. 9608-9616, Sept. 2020. 
[6] X. Sun, Z. Shi, G. Lei, Y. Guo, and J. Zhu, "Analysis and design optimization of a permanent magnet synchronous motor for a campus patrol electric vehicle," IEEE Trans. Veh. Technol., vol. 68, no. 11, pp. 10535-10544, Nov. 2019.

[7] Y. Jiang, W. Xu, C. Mu, and Y. Liu, "Improved deadbeat predictive current control combined sliding mode strategy for PMSM drive system," IEEE Trans. Veh. Technol., vol. 67, no. 1, pp. 251-263, Jan. 2018.

[8] X. Sun, K. Diao, G. Lei, Y. Guo, and J. Zhu, "Real-time HIL emulation for a segmented-rotor switched reluctance motor using a new magnetic equivalent circuit," IEEE Trans. Power Electron., vol. 35, no. 4, pp. 3841-3849, Apr. 2020.

[9] K. Diao, X. Sun, G. Lei, Y. Guo, and J. Zhu, "Multiobjective system level optimization method for switched reluctance motor drive systems using finite element model," IEEE Trans. Ind. Electron., vol. 67, no. 12, pp. 10055-10064, Dec. 2020.

[10] W. Zhang, Y. Xu, Y. Huang and J. Zou, "Reduction of high-frequency vibration noise for dual-branch three-phase permanent magnet synchronous motors," Chinese Journal of Electrical Engineering, vol. 6, no. 2, pp. 42-51, Jun. 2020.

[11] J. Jung, V. Q. Leu, T. D. Do, E. Kim, and H. H. Choi, "Adaptive PID speed control design for permanent magnet synchronous motor drives," IEEE Trans. Power Electron., vol. 30, no. 2, pp. 900-908, Feb. 2015.

[12] X. Sun, C. Hu, G. Lei, Z. Yang, Y. Guo, and J. Zhu, "Speed sensorless control of SPMSM drives for EVs with a binary search algorithm-based phase-locked loop," IEEE Trans. Veh. Technol., vol. 69, no. 5, pp. 4968-4978, May 2020.

[13] R. Cai, R. Zheng, M. Liu, and M. Li, "Robust control of PMSM using geometric model reduction and $\mu$-synthesis," IEEE Trans. Ind. Electron., vol. 65, no. 1, pp. 498-509, Jan. 2018.

[14] H. Chaoui, M. Khayamy, and A. A. Aljarboua, "Adaptive interval type2 fuzzy logic control for PMSM drives with a modified reference frame," IEEE Trans. Ind. Electron., vol. 64, no. 5, pp. 3786-3797, May. 2017.

[15] X. Sun, M. Wu, G. Lei, Y. Guo, and J. Zhu, "An improved model predictive current control for PMSM drives based on current track circle," IEEE Trans. Ind. Electron., 2020, DOI: 10.1109/TIE.2020.2984433.

[16] S. Song, R. Hei, R. Ma and W. Liu, "Model predictive control of switched reluctance starter/generator with torque sharing and compensation," IEEE Trans. Transport. Electrific., vol. 6, no. 4, pp. 1519-1527, Dec. 2020.

[17] X. Sun, C. Hu, G. Lei, Y. Guo, and J. Zhu, "State feedback control for a PM hub motor based on grey wolf optimization algorithm," IEEE Trans. Power Electron., vol. 35, no. 1, pp. 1136-1146, Jan. 2020.

[18] X. Sun, Z. Jin, Y. Cai, Z. Yang, and L. Chen, "Grey wolf optimization algorithm based state feedback control for a bearingless permanent magnet synchronous machine," IEEE Trans. Power Electron., vol. 35, no. 12 , pp. 13631-13640, Dec. 2020.

[19] M. A. M. Cheema, J. E. Fletcher, M. Farshadnia, D. Xiao, and M. F. Rahman, "Combined speed and direct thrust force control of linear permanent-magnet synchronous motors with sensorless speed estimation using a sliding-mode control with integral action," IEEE Trans. Ind. Electron., vol. 64, no. 5, pp. 3489-3501, May. 2017.

[20] X. Sun, J. Cao, G. Lei, Y. Guo, and J. Zhu, "Speed sensorless control for permanent magnet synchronous motors based on finite position set," IEEE Trans. Ind. Electron., vol. 67, no. 7, pp. 6089-6100, Jul. 2020.

[21] W. Xu, A. K. Junejo, Y. Liu, and M. R. Islam, "Improved continuous fast terminal SMCwith extended state observer for speed regulation of PMSM drive system," IEEE Trans. Veh. Technol., vol. 68, no. 11, pp. 10465-10476, Nov. 2019.

[22] V. Repecho, D. Biel, and A. Arias, "Fixed switching period discretetime sliding mode current control of a PMSM," IEEE Trans. Ind. Electron., vol. 65, no. 3, pp. 2039-2048, Mar. 2018.

[23] X. Sun, L. Feng, K. Diao and Z. Yang, "An improved direct instantaneous torque control based on adaptive terminal sliding mode for a segmented-rotor SRM," IEEE Trans. Ind. Electron., doi: 10.1109/TIE.2020.3029463.

[24] J. Liu, H. Li, and Y. Deng, "Torque ripple minimization of PMSM based on robust ILC via adaptive sliding mode control," IEEE Trans. Power Electron., vol. 33, no. 4, pp. 3655-3671, Apr. 2018.

[25] D. Liang, J. Li, R. Qu, and W. Kong, "Adaptive second-order sliding- mode observer for PMSM sensorless control considering VSI nonlinearity," IEEE Trans. Power Electron., vol. 33, no. 10, pp. 89949004 , Oct. 2018.

[26] A. Sabanovic, "Variable structure systems with sliding-modes in motion control-a survey," IEEE Trans. Ind. Informat., vol. 7, no. 2, pp. 212-223, May 2011

[27] F. Lin, Y. Hung, and M. Tsai, "Fault-tolerant control for six-phase PMSM drive system via intelligent complementary sliding-mode control using TSKFNN-AMF," IEEE Trans. Ind. Electron., vol. 60, no. 12, pp. 5747-5762, Dec. 2013.

[28] F. J. Lin, J. C. Hwang, P. H. Chou, and Y. C. Hung, "FPGA-based intelligent-complementary sliding-mode control for PMLSM servodrive system," IEEE Trans. Power Electron., vol. 25, no. 10, pp. $2573-$ 2587, Oct. 2010.

[29] Y. Feng, X. Yu, and F. Han, "High-order terminal sliding-mode observer for parameter estimation of a permanent-magnet synchronous motor," IEEE Trans. Ind. Electron., vol. 60, no. 10, pp. 4272-4280, Oct. 2013.

[30] X. Zhang, L. Sun, K. Zhao, and L. Sun, 'Nonlinear speed control for PMSM system using sliding-mode control and disturbance compensation techniques," IEEE Trans. Power Electron., vol. 28, no. 3, pp. 1358-1365, Mar. 2013.

[31] A. Bartoszewicz and P. Leśniewski, "New switching and nonswitching type reaching laws for SMC of discrete time systems," IEEE Trans. Control Syst. Technol., vol. 24, no. 2, pp. 670-677, Mar. 2016.

[32] C. J. Fallaha, M. Saad, H. Y. Kanaan, and K. Al-Haddad, "Slidingmode robot control with exponential reaching law," IEEE Trans. Ind. Electron., vol. 58, no. 2, pp. 600-610, Feb. 2011.

[33] X. Sun, J. Wu, G. Lei, Y. Guo and J. Zhu, "Torque ripple reduction of SRM drive using improved direct torque control with sliding mode controller and observer," IEEE Trans. Ind. Electron., doi: 10.1109/TIE.2020.3020026.

[34] X. Zhang and Z. Li, "Sliding-mode observer-based mechanical parameter estimation for permanent magnet synchronous motor," IEEE Trans. Power Electron., vol. 31, no. 8, pp. 5732-5745, Aug. 2016.

[35] Y. Li, B. Yang, T. Zheng, Y. Li, M. Cui and S. Peeta, "Extended-StateObserver-Based Double-Loop Integral Sliding-Mode Control of Electronic Throttle Valve," IEEE Trans. Intell. Transp. Syst., vol. 16, no. 5, pp. 2501-2510, Oct. 2015.

[36] W. Gao and J. C. Hung, "Variable structure control of nonlinear systems: a new approach," IEEE Trans. Ind. Electron., vol. 40, no. 1, pp. 45-55, Feb. 1993.

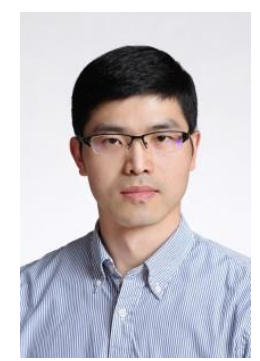

Xiaodong Sun (M'12-SM'18) received the B.Sc. degree in electrical engineering, and the M.Sc. and Ph.D. degrees in control engineering from Jiangsu University, Zhenjiang, China, in 2004, 2008, and 2011, respectively.

Since 2004, he has been with Jiangsu University, where he is currently a Professor in Vehicle Engineering with the Automotive Engineering Research Institute. From 2014 to 2015, he was a Visiting Professor with the School of Electrical, Mechanical, and Mechatronic Systems, University of Technology Sydney, Sydney, Australia. His current teaching and research interests include electrified vehicles, electrical machines, electrical drives, and energy management. He is the author or coauthor of more than 100 refereed technical papers and one book, and he is the holder of 42 patents in his areas of interest. Dr. Sun is an Editor of the IEEE TRANSACTIONS ON ENERGY CONVERSION. 


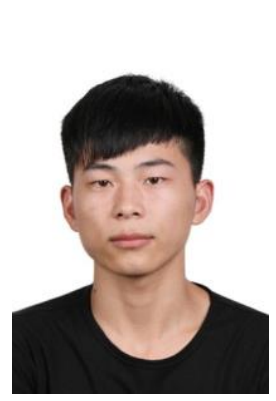

Junhao Cao was born in Huaian, Jiangsu, China, in 1994. He received the B.S. degree in vehicle engineering from Jiangsu University, Zhenjiang, China, in 2018, and he is currently working toward the M.E. degree in Jiangsu University, Zhenjiang, China.

His current research interests include control of electrical drive systems and advanced control strategy of electric machine.

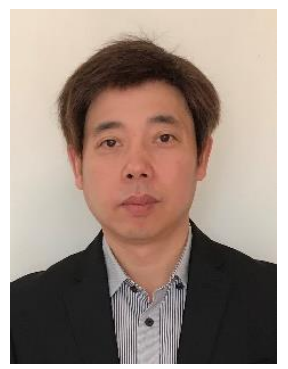

Gang Lei (M'14) received the B.S. degree in Mathematics from Huanggang Normal University, China, in 2003, the M.S. degree in Mathematics and $\mathrm{Ph} . \mathrm{D}$. degree in Electrical Engineering from Huazhong University of Science and Technology, China, in 2006 and 2009, respectively.

$\mathrm{He}$ is currently a Senior Lecturer at the School of Electrical and Data Engineering, University of Technology Sydney (UTS), Australia. His research interests include computational electromagnetics, design optimization and control of electrical drive systems and renewable energy systems. He is an Associate Editor of the IEEE TRANSACTIONS ON INDUSTRIAL ELECTRONICS and an Editor of the IEEE TRANSACTIONS ON ENERGY CONVERSION.

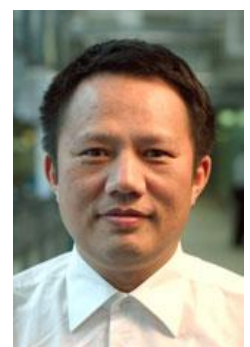

Youguang Guo (S'02-M'05-SM'06) received the B.E. degree from Huazhong University of Science and Technology, China in 1985, the M.E. degree from Zhejiang University, China in 1988, and the Ph.D. degree from University of Technology, Sydney (UTS), Australia in 2004, all in electrical engineering. $\mathrm{He}$ is currently a professor at the School of Electrical and Data Engineering, University of Technology Sydney (UTS). His research fields include measurement and modeling of properties of magnetic materials, numerical analysis of electromagnetic field, electrical machine design optimization, power electronic drives and control.

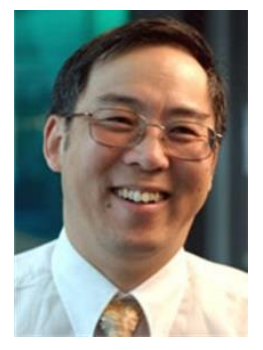

Jianguo Zhu (S'93-M'96-SM'03) received the B.E. degree in 1982 from Jiangsu Institute of Technology, Jiangsu, China, the M.E. degree in 1987 from Shanghai University of Technology, Shanghai, China, and the Ph.D. degree in 1995 from the University of Technology Sydney (UTS), Sydney, Australia, all in electrical engineering. He was appointed a lecturer at UTS in 1994 and promoted to full professor in 2004 and Distinguished Professor of Electrical Engineering in 2017. At UTS, he has held various leadership positions, including the Head of School for School of Electrical, Mechanical and Mechatronic Systems and Director for Centre of Electrical Machines and Power Electronics. In 2018, he joined the University of Sydney, Australia, as a full professor and Head of School for School of Electrical and Information Engineering. His research interests include computational electromagnetics, measurement and modelling of magnetic properties of materials, electrical machines and drives, power electronics, renewable energy systems and smart micro grids. 